\title{
Mosaic mentoring: finding the right mentor for the issue at hand
}

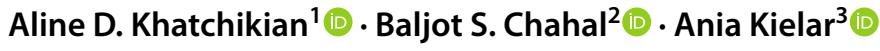 \\ Received: 10 January 2021 / Revised: 4 October 2021 / Accepted: 5 October 2021 / Published online: 30 October 2021 \\ (c) The Author(s), under exclusive licence to Springer Science+Business Media, LLC, part of Springer Nature 2021
}

\begin{abstract}
Mentoring has a vital role throughout the medical profession. Over the past few years, mentoring has become an area of focus as being an important aspect of radiologists' career starting from the early trainee level. A variety of mentorship strategies have come to the forefront, allowing many avenues for those seeking to engage in mentorship as either a mentee or mentor. Mosaic mentoring is a new approach that emphasizes utilizing a collection of mentorship approaches to maximize outcomes based on individual and/or domain-specific needs. The purpose of our paper is to provide a brief overview of a variety of mentorship models while introducing the concept of mosaic mentoring and exploring how it can benefit radiologists throughout their career.
\end{abstract}

Keywords Mentorship $\cdot$ Mosaic mentoring $\cdot$ Radiology education $\cdot$ Medical education $\cdot$ Early career

\section{Introduction}

The etymology of the word mentor provides a strong basis upon which to understand its importance. It originated from Greek mythology and the epic poem, Odyssey. Odysseus was said to have entrusted his son Telemachus with his friend mentor, who offered the latter his guidance and support. The concept of mentorship has since been integrated into modern society and in particular within the medical profession, given that teaching is a fundamental component of the Hippocratic Oath [1].

The evolution of medical mentorship has become more commonplace with regards to domain-specific initiatives. With the increasing complexity of our profession and its myriad academic, professional, as well as personal obligations, there is a greater emphasis on mentoring in radiology

Ania Kielar

ania.kielar@uhn.ca

1 Department of Diagnostic Radiology, McGill University, Montreal General Hospital Site, 1650 Cedar Avenue, Rm C5 118, Montreal, QC H3G 1A4, Canada

2 Department of Radiology and Diagnostic Imaging, 2A2.41 WC Mackenzie Health, Sciences Centre, 8440112 Street NW, Edmonton, AB T6G 2B7, Canada

3 Department of Medical Imaging, Toronto General Hospital, 585 University Avenue, Toronto, ON MG5 2N2, Canada than ever before. Guidance through one's medical training and early career can facilitate optimal achievement of academic and personal milestones. Mosaic mentoring is a concept that encourages mentees to integrate various mentorship models and strategies in order to ensure the best mentor and format is selected for the issue at hand while recognizing the evolution of their needs over time.

\section{Defining mentorship models and mosaic mentorship}

Mentorship takes place when an individual, typically one more experienced in a given domain, provides advice and support to a mentee as part of a formal or informal rapport, based on mutual consent. Mentorship is separate although often referred to with multiple other types of professional interactions, including sponsorship, coaching, and role modeling (Table 1).

Mentorship can be categorized into different models based on the nature of the connections between individuals. Formal mentorship in a residency or medical school program is generally targeted toward specific domains (e.g., research and clinical) and is integrated into department-supported activities. It enforces accountability from mentors and mentees and typically involves departmental incentives, such as funding for various interactive opportunities. However, as 
Table 1 Definitions of mentorship and associated terms

Term

Mentorship

An individual, typically more experienced in a given domain, provides advice and support to a mentee as part of an informal or formal connection based on mutual consent

Sponsorship

When an individual, typically in a position of power or authority, uses their social capital to advocate on behalf of another person. This can involve placing the reputation of the sponsor on the line

Coaching A performance and goal-oriented process designed to achieve specific outcomes or metrics

Role modeling

An individual serves as an exemplar of positive (or negative) behaviors for another individual to emulate or avoid

it is an organized system where mentors and mentees are typically matched by someone else, these relationships are not created organically and may not meet all of the mentee's needs. In contrast, informal mentorship is unstructured without formal integration into workplace responsibilities. Informal mentorship can allow mentees and mentors to selfselect based on personality or other compatibilities. However, informal mentorship may lack specific accountability as well as posing potential barriers for individuals who consider themselves more introverted, as they may experience challenges in reaching out to potential informal mentors.

Mentorship can also be classified based on the number of people involved in the interactions. One-on-one mentoring involves only one mentor and one mentee. Group or teambased mentoring is an alternative option that allows one or multiple mentors to guide several mentees at once or in tandem. This can allow for flexibility in scheduling and provide a diversity of meeting opinions. Geographical challenges as well as current social distancing requirements between a mentor and mentee can be overcome with "distance mentoring" including over the phone interactions or via videoconferencing. A self-mentoring model has also been described, whereby the mentee acts as their own mentor, using forms of self-reflection or external written and video materials as tools5. Self-mentoring can potentially empower oneself and lead to increased communication skills and self-confidence which in turn can improve leadership and organizational skills [2]. To date, there are no specific publications about the outcomes of self-mentoring specifically in radiology. However, intentional motivational and leadership language have been validated to be a strategic medium for leaders in the USA and internationally to positively influence their peers [2]. This strategic communication medium can be adapted toward achieving personal goals and advancing one's radiology career.

Another mentorship strategy that can be highly effective for radiologists in training and staff radiologists is peer-topeer mentorship. This includes colleagues at the same stage of their career, for example, two residents in the same year of training. Near-peer mentoring reflects interactions between individuals in close career stage, for example, between a senior and a junior resident 3 . Peer-to-peer mentoring has the potential to remove a mentees' perceived fear of negative evaluation from mentors that could potentially arise in more traditional hierarchical interactions [3]. In comparison, reverse mentoring is an innovative mentorship model that connects younger mentors with older mentees to share knowledge and experiences on cultural and technological advances. This model reverses the traditional perception of mentors and mentees and allows for a two-way process of exchanging experiences and ideas [4].

Based on the various mentorship models described above, the concept of mosaic mentoring has emerged (Fig. 1). Mosaic mentoring allows the mentee to benefit from multiple mentors as well as from various mentorship strategies based on their individualized needs, at a given time in their journey through radiology, while also exploring a conglomerate of learning strategies. Given the complexity of radiology careers, both academics, community, and private practice, the mosaic model allows for flexibility and comprehensive mentorship options.

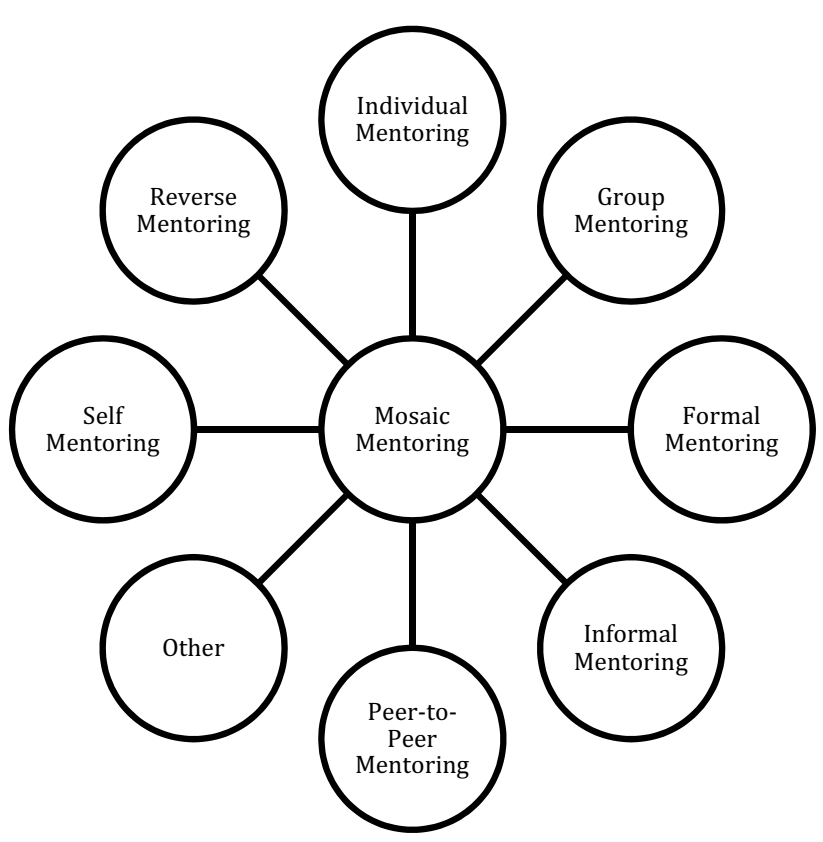

Fig. 1 Components of mentoring that can comprise the mosaic mentorship model 


\section{The evolution of mentorship needs in radiology}

As a mentee progresses in their career, their needs evolve and change. Self-reflection and dynamic goal setting are central to successful mentorship experiences at all stages of training, from medical school to residency, as well as into early career and even in established career stages. Selecting the right mentor or mentors is a key component of mosaic mentorship. Data have shown that there is increased mentee satisfaction when mentors are chosen by mentees, rather than being assigned [5]. Studies have also demonstrated that female residents and female junior staff preferred and benefited most, from female mentors [6]. There is lack of data on the benefits of ethnically diverse mentors, specifically from underrepresented racial and ethnic minorities in radiology, including indigenous populations. This is in line with the paucity of research on ethnic and racial diversity and disparities in radiology. The lack of underrepresented racial and ethnic minority role models may potentially contribute to lack of diversity in radiology as per Lebel et al. [7]. If radiology departments were to develop mentorship opportunities for women and visible minority medical students, this would provide early exposure to the field and improve overall diversity in Canadian radiology practices and leadership [7].

\section{Medical students}

Having a mentor in radiology allows medical students to get acquainted with the value of radiology in medicine and become familiar with the tasks as well as experiences of radiology residents, fellows, and radiologists. Radiologyspecific mentorship has been demonstrated to increase the likelihood of medical students pursuing a career in radiology [8]. Early mentorship should be encouraged in medical schools given that recent trends in the USA have demonstrated that fewer medical students are choosing radiology as a career $[9,10]$.

Mosaic mentorship can allow medical students to be exposed to various viewpoints so that they can make informed decisions regarding their future career directions. Having mentors allows learners to identify nuances related to issues around medical school academic performance, prerequisites to residency admission, fellowships choices, and job opportunities [11]. Many medical schools already offer formal mentorship programs by matching medical students to mentors. Informal mentoring can be achieved by having potential mentors engage with medical student radiology interest groups, as this has been shown to be perceived as a valuable opportunity for mentorship by medical students [12].

\section{Radiology residents and fellows}

Mentoring programs with various models also exist in radiology training programs. A recent study which surveyed 70 of the 156 members of the Association of Program Directors in Radiology confirmed that $57 \%$ of residency training program offered a mentorship program6. One of the benefits of these formal mentorship programs is to teach residents how to become future mentors, thereby allowing subsequent organic development of mentorship opportunities. Mentorship opportunities are also promoted by resident and fellow sections which exist for nearly all radiology organizations. This outreach has also recently expanded to include medical students. Annual meetings have become well established as a means of creating and reinforcing mentor relationships. Several organizations have established speed mentoring sessions at their annual meeting allowing trainees to rapidly meet mentors from across the nation which they potentially would not have been able to interact with otherwise. Some meetings, such as the ACR Annual Meeting, have also established mentorship programs dedicated to first-time meeting attendees.

Peer-to-peer mentorship lends itself well to informal pairings developing between colleagues at similar stages of their training or with similar interests. This can be very beneficial in providing relevant advice to mentees. For example, the COVID-19 pandemic resulted in significant modifications to radiology training $[13,14]$. It could be hypothesized that it would be more beneficial for a junior trainee to seek advice regarding dealing with the implications of the pandemic on their training from their immediate seniors rather than someone 20 to 30 years into their career. Likewise, a trainee transitioning out of an administrative or leadership role such as chief resident would be more optimally positioned to provide advice on how to be an effective future chief resident to a mentee, rather than someone who had occupied the role decades prior.

\section{Early-career radiologists}

Early-career radiologists have their own needs for mentorship, sponsorship, and comradery; however, few formal systems are in place for this cohort once they complete their training. Numerous organizations have established early-career committees, including the Society of Abdominal Radiology, to recognize the needs of early-career radiologists. For an early-career radiologist to optimally benefit from mentorship, specific goals should be identified so that the right mentor can be identified. This is even more critical when considering a sponsor as this individual will help 
advance one's career by opening doors in areas where they have influence or connections. Identifying potential mentors inside and outside the workplace can also be of significant importance in order to provide mentorship regarding worklife balance and other personal factors. Mentors outside of the workplace may also have the added benefit of allowing mentees to feel more comfortable showing vulnerability without concerns for negative impacts at work.

Peer-to-peer mentorship also has a role to play in groups with more than one new staff or in small radiology groups. In academic centers, mentoring needs for early-career radiologists may include obtaining guidance for how to efficiently start projects as the primary investigator, how to identify what projects to work on, and how to effectively oversee trainees in one's research projects. Additional benefits include learning tips and tricks for early-career advancement, including being shown good habits, such as keeping one's curriculum vitae up to date, volunteering for committees, and completing administrative tasks on time. In community or private practice, there are also many opportunities for career advancement with associated opportunities for mentorship. Examples include getting started in public health and community practice-related translational research, becoming involved in radiology organizations, and finding ways to become efficient with workloads in highvolume practices.

\section{Implementing mosaic mentoring}

What makes mosaic mentoring unique is its ability to adapt at every training and career milestone. It can be implemented in a plethora of ways in accordance with the priorities and goals of the mentee. Medical students can get start getting involved in mosaic mentoring by approaching professors during radiology lectures and asking for a meeting to learn more about their career. This can progress into a shadowing opportunity in their workplace and an introduction to additional radiology colleagues. This informal model can help a student find a research supervisor which would further advance their interest in radiology. A more formal mosaic mentoring program can also be initiated by contacting a department's radiology program director and administrator and asking to be linked with one or multiple radiologists. As for residents and fellows, one can get involved in mosaic mentoring by the means of their program's formal mentor matching program, if applicable, or by directly approaching staff who lead careers they admire. If there is no formal program, it can be created with the help of radiology chief residents, residency, and fellowship directors. Trainees can meet additional mentors at conferences to learn about different ways to lead a successful radiology practice. In early career, mosaic mentoring can be integrated by informally meeting with colleagues of similar seniority to discuss shared career and personal hurdles. A more formal mentorship program can be set with senior radiologists of similar or different institutions to seek advice on career-specific milestones just as research grant applications and academic promotions.

For all stages of one's career, social media have become a potentially strong development platform for both informal and formal mentorship programs [15]. Most recently, programs have been established through Twitter accounts to create mentorship match programs in radiology. This allowed for hundreds of mentors to be virtually matched across the globe during the COVID-19 pandemic, addressing a huge need amid travel restrictions and conference cancelations. In addition, social media have been utilized to create mentorship opportunities for women and underrepresented minorities, groups that in the past have had difficulty accessing mentorships in radiology $[3,15,16]$.

Positive results from mentorship programs have been shown in a study by Bredella et al. In the study published in March 2021, after implementation of a mentorship program, the mentees who were enrolled had higher rates of promotion when compared to the previous 5 years [17]. In addition, multiple mentees were recognized with awards and winning grants [17]. The multiple benefits of mosaic mentoring out way its pitfalls. However, to maximize the positive impact of a hybrid model of mentorship styles, mentees must have clear goals and timelines in order to avoid unmet expectations and optimize time spent with mentors. Implementing specific, measurable, attainable, relevant, and time-bound (SMART) objectives can help achieve this step [18].

\section{Conclusion: the value of mosaic mentoring}

Mentorship has taken on increased importance in the radiology community as it progressively becomes recognized as a means to help maximize both individual and organizational outcomes. A variety of mentorship strategies and models exist including formal and informal strategies, individual and group-based formats, as well as peer-to-peer, self-mentoring, hierarchical, and reverse models. Each strategy has its unique benefits and potential pitfalls, thereby increasing the importance of a hybrid approach, coined "mosaic mentoring." By utilizing mosaic mentoring, early-career radiologists and trainees can ensure they select the appropriate mentor for the given domain while optimizing their chances of finding success in their mentoring relationships.

Acknowledgements None.

\section{Declarations}

Conflict of interest The authors declare that they have no conflict of interest. 


\section{References}

1. Ambrosetti A, Davis S. Mentor. M/C J. 2016;19(2). https://doi. org/10.5204/mcj. 1103

2. Holmes WT, Carr M. Motivating language and self-mentoring: a training program supporting the development of leaders in organizations. Dev Learn Organ. 2017;31(5). https://doi.org/10.1108/ DLO-02-2017-0010

3. Thomas N, Bystydzienski J, Desai A. Changing Institutional Culture through Peer Mentoring of Women STEM Faculty. Innov High Educ. 2014;40(2). https://doi.org/10.1007/ s10755-014-9300-9

4. Clarke AJ, Burgess A, van Diggele C, Mellis C. $<\mathrm{p}>$ The role of reverse mentoring in medical education: current insights $</ \mathrm{p}>$. Adv Med Educ Pract. 2019; Volume 10. https://doi.org/10.2147/ amep.s179303

5. Yamada K, Slanetz PJ, Boiselle PM. Perceived benefits of a radiology resident mentoring program: Comparison of residents with self-selected Vs assigned mentors. Can Assoc Radiol J. 2014;65(2). https://doi.org/10.1016/j.carj.2013.04.001

6. Donovan A. Views of radiology program directors on the role of mentorship in the training of radiology residents. Am J Roentgenol. 2010;194(3). https://doi.org/10.2214/AJR.09.3403

7. Lebel K, Hillier E, Spalluto LB, Yap W, Keglowitsch K, Darras KE, Yong-Hing CJ. The Status of Diversity in Canadian Radiology-Where We Stand and What Can We Do About It. Can Assoc Radiol J. 2020 Dec 22:846537120978258. https://doi.org/10.1177/ 0846537120978258 . Epub ahead of print. PMID: 33353386.

8. Yoon JD, Ham SA, Reddy ST, Curlin FA. Role Models' Influence on Specialty Choice for Residency Training: A National Longitudinal Study. J Grad Med Educ. 2018;10(2). https://doi.org/10. 4300/JGME-D-17-00063.1

9. Hoffmann JC, Flug JA. A Call to Action for Medical Student Mentoring by Young Radiologists. Curr Probl Diagn Radiol. 2016;45(2). https://doi.org/10.1067/j.cpradiol.2015.08.002

10. Sit C, Srinivasan R, Amlani A, et al. Attitudes and perceptions of UK medical students towards artificial intelligence and radiology: a multicentre survey. Insights Imaging. 2020;11(1). https://doi. org/10.1186/s13244-019-0830-7
11. Amorosa JK. How Do I Mentor Medical Students Interested in Radiology? Acad Radiol. 2004;11(1). https://doi.org/10.1016/ S1076-6332(03)00475-6

12. Dobson JL, Fenwick A, Linehan V, Hartery A. Radiology Interest Groups: A Recipe for Success. Can Assoc Radiol J. Published online 2020. https://doi.org/10.1177/0846537119899551

13. Odedra D, Chahal BS, Patlas MN. Impact of COVID-19 on Canadian Radiology Residency Training Programs. Can Assoc Radiol J. 2020;71(4). https://doi.org/10.1177/0846537120933215

14. Chertoff JD, Zarzour JG, Morgan DE, Lewis PJ, Canon CL, Harvey JA. The Early Influence and Effects of the Coronavirus Disease 2019 (COVID-19) Pandemic on Resident Education and Adaptations. J Am Coll Radiol. 2020;17(10). https://doi.org/10. 1016/j.jacr.2020.07.022

15. Spieler B, Ballard DH, Mazaheri P, et al. Social Media in Radiology: Overview and Usefulness of Online Professional \#SoMe Profiles. Acad Radiol. Published online 2020. https://doi.org/10. 1016/j.acra.2020.03.014

16. Retrouvey M, Keefe B, Kotsenas A, McGinty G, Patel AK. Women in Radiology: Creating a Global Mentorship Network Through Social Media. J Am Coll Radiol. 2018;15(1). https:// doi.org/10.1016/j.jacr.2017.09.029

17. Bredella MA, Alvarez C, O'Shaughnessy SA, Lavigne SD, Brink JA, Thrall JH. Radiology Mentoring Program for Early Career Faculty-Implementation and Outcomes. J Am Coll Radiol. 2021 Mar;18(3 Pt A):451-456. https://doi.org/10.1016/j.jacr.2020.09. 025. Epub 2020 Oct 5. PMID: 33031784; PMCID: PMC7935755.

18. Bjerke MB, Renger R. Being smart about writing SMART objectives. Eval Program Plann. 2017 Apr;61:125-127. https://doi.org/ 10.1016/j.evalprogplan.2016.12.009. Epub 2016 Dec 23. PMID: 28056403 .

Publisher's Note Springer Nature remains neutral with regard to jurisdictional claims in published maps and institutional affiliations. 